\title{
Productos botánicos para el manejo del ácaro blanco (Polyphagotarsonemus latus, Banks.) (Acarina; Tarsonemidae), en chiltoma (Capsicum annuum L.), en Masaya, Nicaragua
}

\section{Botanical insecticides to control the broad mite (Polyphagotarsonemus latus, Banks.) (Acarina; Tarsonemidae), in green pepper (Capsicum annuum L.), in Masaya, Nicaragua}

\section{Edgardo Jiménez-Martínez ${ }^{1}$, Ariel Mena-García ${ }^{2}$, Ilda Rayo-Cruz ${ }^{2}$}

${ }^{1}$ Facultad de Agronomía, Universidad Nacional Agraria, edgardo.jimenez@ci.una.edu.ni, 505-2263-2609

${ }^{2}$ Graduados de la carrera de Ingeniería Agronómica, Universidad Nacional Agraria

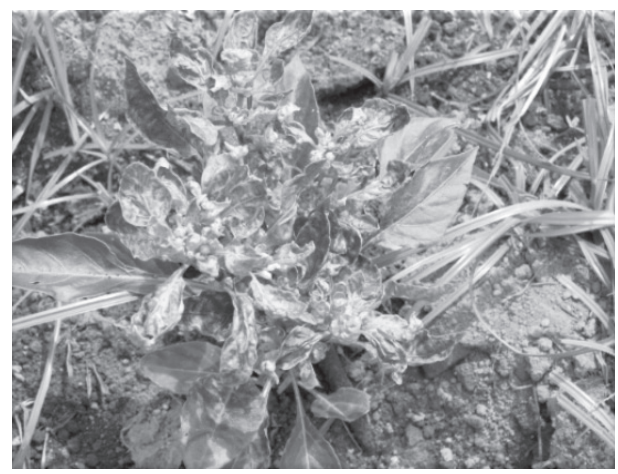

\section{RESUMEN}

Con el objetivo de evaluar cinco productos botánicos para el manejo del ácaro blanco de la chiltoma, se realizó un estudio en el Municipio de Tisma, Masaya en el período comprendido de Septiembre a Noviembre del año 2014. Las alternativas evaluadas fueron: Eucalipto, Neem, Crisantemo, Madero Negro y Chile+Ajo+Jabon en comparación con el testigo que fue solamente aplicación de agua. Las variables evaluadas fueron: Número de ácaro blanco por planta, porcentaje de severidad del daño de ácaro blanco por planta, además de algunas variables económicas como el rendimiento en $\mathrm{kg}$ / ha por tratamiento evaluado, análisis de presupuesto parcial, análisis de dominancia y tasa de retorno marginal. Los resultados obtenidos en el estudio determinan que los tratamientos Madero Negro y chile+ajo+jabon fueron los que presentaron el mejor efecto de control del ácaro blanco, los mejores rendimientos comerciales lo obtuvieron los tratamientos madero negro y chile+ajo+jabon, las mejores tasas de retorno marginal fueron obtenidas en los tratamientos madero negro seguido del chile+ajo+jabon.

Palabras clave: acaro blanco, insecticidas botánicos, chiltoma, pimiento dulce.

\section{ABSTRACT}

In order to evaluate five botanicals insecticides for the management of the green pepper white mite, a study was conducted in the municipality of Tisma, Masaya in the period from September to November 2014. The evaluated products were: Eucalyptus, Neem, Chrysanthemum, Madero negro and Chili pepper + Garlic+Soap compared with the control treatment which consisted in water only. The variables evaluated were: number of white mites per plant, severity damage percent of white mite per plant, plus some economic variables as the yield in $\mathrm{kg} / \mathrm{ha}$, partial budget analysis, dominance analysis and marginal rate of return. The results of the study determined that treatments Madero negro and chili pepper+garlic+soap were those with the best control effect of white mites, the best commercial returns were obtained by madero negro followed by chili pepper+garlic+soap, top marginal rates of return were obtained in the treatments madero negro followed by Chili pepper+garlic+soap.

Key words: White mite, botanical insecticides, green pepper. 
$\mathrm{L}$ a chiltoma Capsicum annuum L. (Orden Polemoniales, Familia solanácea), es una hortaliza bien importante por su valor nutritivo, es rica en vitaminas A, B1, B2 y C (INTA, 2004). Es una planta originaria de regiones tropicales específicamente de Bolivia y Perú. De estos países se propagó para la mayor parte de los países del continente americano, se adaptó en las diferentes regiones como el sur de Brasil, México y luego en el resto del mundo (CATIE, 1993), este cultivo se desarrolla en muchos tipos de suelos desde arcillosos hasta arenosos, limosos y lo hace con éxito en suelo con pH en un rango de 5.0 a 6.5 (Sevilla y Rodríguez, 2009).

En Nicaragua, se estima que el área que se cultiva anualmente es de 1,070 hectáreas, localizándose casi la mitad de la producción en el Valle de Sébaco (Matagalpa), con rendimientos promedios de $15 \mathrm{t} / \mathrm{ha}$. Otras regiones donde se siembra este cultivo a pequeña escala son: Ocotal, Somoto, Estelí, Jinotega, Matagalpa, Boaco, Granada, Masaya específicamente en Tisma (INTA, 2006; Martínez y Jirón, 2011).

En el departamento de Masaya la chiltoma se siembra mayormente en el municipio de Tisma, con un área cultivada que se estima en unas 25 hectáreas, muchas áreas de este municipio se encuentran altamente contaminadas con plaguicidas químicos, esto debido a que el $95 \%$ de los agricultores de la zona desde hace muchos años han hecho uso excesivo de los pesticidas químicos para control de organismos plagas, los cuales han creado resistencia a estos productos y esto ha repercutido con efectos negativos como: bajos rendimientos, eliminación de la fauna benéfica (arañas, mariquitas, hormigas, etc.), afectaciones a la salud humana y al medio ambiente. El uso incorrecto de los productos químicos sintéticos ha generado desequilibrio en el agroecosistema, provocando que organismos que antes se consideraban como plagas secundarias se conviertan en plagas primarias (González y Obregón, 2007).

Este rubro es cultivado principalmente por los pequeños y medianos productores, quienes siembran parcelas de 0.3 hectárea, hasta áreas de tres o cuatro hectáreas, en un sistema de monocultivo, destinadas para los mercados locales (INTA, 2006). Este cultivo está expuesto a diferentes factores que inciden en el rendimiento de cada cosecha, como diferentes tipos de enfermedades, virosis, tizones y manchas bacterianas. En cuanto a insectos plagas, se puede mencionar a mosca blanca, picudos, minador de hojas, áfidos y actualmente el ácaro blanco. Este último, en el municipio de Tisma, está ocasionando daños muy severos en las plantaciones de chiltoma (González y Obregón, 2007; INTA, 2004).

El ácaro blanco, Poliphagotarsonemus latus, Banks, (Acarina; Tarsonemidae), se considera como el ácaro de mayor importancia en Centroamérica. En Nicaragua es la segunda plaga de mayor importancia en el cultivo de chiltoma y en los últimos tiempos ha llegado a ocasionar pérdidas hasta de un cien por ciento (INTA, 2004). Marrón De
Jones (1983), describe que la especie Poliphagotarsonemus latus Banks está compuesta por las siguiente palabras; poly (muchos), phago (alimento), tarsonemus (fisura en el cuarto par de patas), latus (designación del color). El ciclo completo del ácaro blanco es muy rápido, de 4 a 10 días dependiendo de las condiciones de humedad relativa y de temperatura. $P$. latus, se localiza en las hojas tiernas o rebrotes, a lo largo de las venas. Los huevos son de color blanco, translucidos y con óvalos en su interior alineados de forma simétrica; se encuentran adheridos, en áreas escondidas o que presentan hundimientos, de tal manera que queden protegidos (Ochoa, et al., 1991).

El daño que causa en las plantas son, encartuchamiento y doblamiento hacia el envés con la lámina mostrando una apariencia corrugada, las plantas pueden paralizar su desarrollo, exhibiendo una apariencia de enanismo, en algunos casos se aprecia una pérdida parcial de la lámina de la hoja (Ochoa, et al., 1991). Los ácaros se diseminan rápidamente en épocas calurosas y secas, su principal método de dispersión es por el viento. Para identificar esta plaga y poder manejarla es necesario conocer que no se puede observar a simple vista debido a que su tamaño es muy diminuto, que va de 0.1 a $0.2 \mathrm{~mm}$ (INTA, 2004).

En los últimos tres años (2010-2013) en la zona de Tisma, el cultivo de la chiltoma sigue siendo atacada por dicho acaro, ocasionando daños muy severos en los rendimientos de hasta un cincuenta por ciento (Barberena y Lacayo 2011), ante esta situación se pretende conocer si algunos productos botánicos son eficaces para el manejo de esta plaga y con estos resultados poder brindar información sobre algunas alternativas botánicas que sean económicamente rentables pero además que sean amigables con el medio ambiente.

\section{MATERIALES Y MÉTODOS}

Ubicación del área de estudio. El estudio se realizó en el municipio de Tisma departamento de Masaya en el período de Septiembre de 2014 a Noviembre de 2014. Este municipio, está ubicado en la parte noroeste de la capital Managua a una distancia de $36 \mathrm{~km}$, entre las coordenadas $12^{\circ} 04^{\prime}$ latitud norte y $86^{\circ} 01^{\prime}$ longitud oeste y posee una superficie de $126.17 \mathrm{~km} 2$ con una población de 12,697 habitantes, donde el $71 \%$ de la población es rural y el $29 \%$ es urbana, Tisma se encuentra a una altura de $50 \mathrm{msnm}$ y presenta un clima que se caracteriza por ser tropical de sabana, con temperaturas promedios de $27.5^{\circ} \mathrm{C}$ y con precipitaciones pluviales anuales que oscilan entre los 1200 y 1400 mm (AMUNIC, 2005).

Diseño experimental. El estudio se estableció como un experimento en diseño de bloques completos al azar (BCA) con cuatro repeticiones y seis tratamientos. El tamaño de cada parcela correspondió a $6 \mathrm{~m}$ de largo por $6 \mathrm{~m}$ de ancho con un área de $36 \mathrm{~m}^{2}$ por cada tratamiento, formando un área de $216 \mathrm{~m}^{2}$ por bloque, siendo el área total del ensayo de $864 \mathrm{~m}^{2}$. 
Productos botánicos evaluados Eucalipto (Eucalyptus globulu, Labill). Fam. Myrtáceas, su principal componente del aceite esencial es el éter óxido terpénicocineol o eucaliptol, constituyendo el 70-80\%, además contiene hidrocarburos monoterpénicos, hidrocarburos sesquiterpénicos, alcoholes monoterpénicos, alcohol sesquiterpénico y aldehídos alifáticos. El eucalipto es un insecticida de contacto e ingestión y actúa como repelente ante los insectos (Aguirre y Delgado. 2010). La dosis utilizada fue de 454 gramos de hojas de eucalipto molido, disuelto en un litro de agua, con 24 horas de reposo, diluido en 20 litros de agua (Jiménez-Martínez y Varela, 2013).

Neem (Azaridachta indica, A. Juss.). Fam. Meliaceae, contiene varias sustancias activas entre ellas Azadirachtin, salanin, melantriol, las cuales se encuentran con mayor concentración en la semilla de este. Estas sustancias tienen acción de insecticida repelente, efecto anti alimentario, inhibidor del crecimiento y ovoposición, esterilizante, además acción fungicida y nematicida (Jiménez-Martínez y Varela, 2013). La dosis empleada fue de $10 \mathrm{ml}$ por litro de agua.

Crisantemo (Chrysanthemum cinerariaefolium, Trevir). Fam. Asteraceae, su principal sustancia activa es la piretrina. El piretro es un insecticida de contacto obtenido de sus flores; los componentes de esta planta con actividad insecticida son seis esteres formados por la combinación de ácidos crisantemicos y pirétricos además los alcoholes piretrolona, cinerolona y jasmolona. El modo de acción consiste en que los compuestos extraídos de las flores actúan sobre el sistema nervioso central y el sistema nervioso periférico de los insectos. Penetran la cutícula del insecto hasta llegar a los centros nerviosos. (Jiménez-Martínez y Varela, 2013). Se utilizó una dosis de 908 gramos de flores y tallos de crisantemo molidos y disueltos en un litro de agua, con 24 horas de reposo, por bomba de 20 litros (Jiménez-Martínez y Varela, 2013).

Madero negro (Gliricidia sepium Jacq). Fam. Fabaceae, es un insecticida y abono foliar que contiene flavonoides, su toxicidad se debe a la conversión por las bacterias de cumarinas a dicoumerol durante la fermentación (Lanuza y Rizo, 2012). Es un insecticida de contacto e ingestión, además actúa como repelente ante los insectos (Jiménez-Martínez y Varela, 2013). La dosis utilizada fue de 454 gramos de hojas de madero negro molido y disuelto en un litro de agua, con 24 horas de reposo, por bomba de 20 litros (Jiménez-Martínez y Varela, 2013).

Chile (Capsicum chinense) Fam. Solanáceas + Ajo (Allium sativum L.) Amaryllidaceae + Jabón (detergente xedex). El chile contiene una sustancia de pungencia elevada llamada capsicina que al ser aplicada sobre los insectos plaga genera una sensación de ardor en todo su cuerpo, por lo cual los insectos dejan de alimentarse, huyen del lugar y mueren. (Ji-
ménez-Martínez y Varela, 2013). El Ajo contiene compuestos de azufre (tiosulfatos) los cuales sobre excitan el sistema nervioso de los insectos y ácaros produciendo irritación, desorientación y repelencia (Jiménez-Martínez y Varela, 2013). El detergente actúa como adherente al follaje, cuando entra en contacto con los tejidos grasosos del ácaro los mata por deshidratación (Martínez y Jirón, 2011). La dosis utilizada fue de 100 gramos de chile, 28 gramos de detergente y una cabeza de ajo, molido y disuelto en un litro de agua, con 24 horas de reposo, por bomba de 20 litros (Jiménez-Martínez y Varela, 2013).

Testigo. En este tratamiento se aplicó agua solamente.

Descripción del estudio (preparación de semillero y manejo). El estudio se basó en el establecimiento de un semillero bajo condiciones de micro invernadero; se utilizó la variedad Nathalie la cual se caracteriza por tener fruto alargado, terminado en punta sin hombros, paredes gruesas, excelente color y sabor, peso de $170 \mathrm{~g}$ en promedio; alto cuajado de flores, tolerancia a phytopthora, TMV, TVY y TVE (Syngenta, 2015); manejando 13 bandejas de polietileno de 105 celdas, con un sustrato de coco molido, éstas fueron tratadas con fungicida Phyton $\AA$. El trasplante al campo se llevó a cabo a los 35 días después de establecido el semillero. La preparación del terreno fue dos pases de arado y uno de grada, la distancia de siembra utilizada fue de $0.5 \mathrm{~m}$ entre plantas y $1.40 \mathrm{~m}$ entre surcos, para un total 17 surcos, 72 plantas por surco, siendo un total de 1224 plantas por parcela.

Muestreos. Para determinar el momento de aplicación de cada uno de los tratamientos los muestreos se realizaron semanalmente desde los $7 \mathrm{ddt}$ hasta los $78 \mathrm{ddt}$, por la mañana y de forma directa para muestrear adultos de ácaro blanco, utilizando lupa de 20X CODDINGTON BAUSH \& LOMB. Para la obtención de los datos se seleccionaron cinco puntos al azar por parcela, en cada punto se tomaron cinco plantas, para un total de 25 plantas muestreadas por parcela y 600 plantas en todo el experimento; se muestrearon específicamente los brotes tiernos.

Aplicaciones. Las aplicaciones de los tratamientos se realizaron cuando se encontró un nivel poblacional promedio de un ácaro blanco por planta. Se utilizó bomba de mochila Jacto ${ }^{\circledR}$ con capacidad de 20 litros de agua.

\section{Variables evaluadas}

Número de ácaro blanco por planta. Esta variable se comenzó a tomar desde los siete días después del trasplante realizando muestreos semanales hasta los71días después del trasplante, donde se muestrearon todos los brotes nuevos de las plantas específicamente el envés de las hoja, lugar donde se encuentran más frecuente el ácaro (Ochoa, et al., 1991). 
Porcentaje de incidencia y severidad del daño de ácaro blanco por planta. La incidencia es la relación de las plantas que presentan síntomas de daño del ácaro blanco con relación al número total de las plantas muestreadas multiplicadas por cien la severidad es el porcentaje de tejido visible dañado o afectado de una planta con relación al total evaluado. Para diferenciar una planta sana de una enferma se realiza a través de la observación del síntoma característico que presentan las hojas de las plantas afectadas por el ácaro, como es la clorosis y deformación de las hojas y ramas (Martínez y Jirón, 2011).

Para determinar la incidencia y severidad del daño del ácaro se realizó toma de datos desde los 7 días hasta los 78 días después del trasplante. Con respecto a la obtención del grado porcentual de la incidencia se utilizó la siguiente fórmula:

$$
\text { Incidencia }(\%)=\frac{\text { Total de plantas con ácaro blanco }}{\text { Número de plantas nuesteadas }} \times 100
$$

Para determinar el grado de severidad ocasionado por el ácaro blanco se usó la escala de severidad utilizada en estudios anteriores por Jiménez, et al, 2013. La escala considera grado 0 , si no hay poresencia de sitomas, grado 1, Débil encrespado hacia arriba en la lámina foliar de hojas nuevas y brotes nuevos, grado 2, Ondulación en hojas nuevas y viejas, grado tres, Encrespado hacia Arriba y deformación en la nervadura central en forma de Zigzag.

Para obtener el grado porcentual de la severidad se utilizó la fórmula general planteada por Vanderplank, 1963.

$$
\text { Severidad }(\%)=\frac{\sum \mathrm{i}}{\mathrm{N}(\operatorname{Vmax})} \times 100
$$

Dónde:

S: Porcentaje de severidad.

$\sum$ i: Sumatoria de datos en plantas muestreadas.

$\mathrm{N}$ : Número de plantas muestreadas.

Vmax: Valor máximo de la escala.

Número y tipo de organismo plaga por planta. Además de las poblaciones de ácaro blanco, simultáneamente se tomaron datos de la ocurrencia poblacional de insectos plagas asociados al cultivo de la chiltoma.

Número y tipo de organismo benéfico por planta. Además de las poblaciones de ácaro blanco y otros organismos plagas, también se tomaron datos de la ocurrencia poblacional de insectos benéficos asociados al cultivo de la chiltoma.

Rendimiento. Para obtener los datos de rendimiento por hectárea se efectuó un solo corte a los 78 ddt, debido al alto porcentaje de severidad de daño ocasionado por el ácaro blanco, Se seleccionaron 10 plantas al azar por parcela, se cosecharon frutos verdes y maduros, se pesó el total de cada parcela de los tratamientos evaluados, se contabilizaron los botones florales para realizar un estimado de cosecha para cada uno de los tratamiento evaluados, para obtener el peso en $\mathrm{kg} \mathrm{ha}^{-1}$.

Análisis económico. Los resultados agronómicos que se obtuvieron del experimento de campo fueron sometidos a análisis económicos, con el propósito de determinar la rentabilidad de los tratamientos en comparación con la práctica común de los productores, o simplemente determinar el tratamiento con mejor retorno económico, todo tratamiento recomendado en la producción deberá ajustarse a los objetivos y circunstancias de los productores.

Se tomaron los datos de rendimientos promedio $(\mathrm{R} \chi)$ por tratamiento y se obtuvo el rendimiento ajustado (Rajust $=10 \%$ de $\mathrm{R} \chi$ ), luego se calculó el beneficio bruto multiplicando el Rajust por el precio de venta de campo $1.90 \mathrm{US} \$ / \mathrm{kg}$. Para la sumatoria de los costos totales que varían, se estimó los costos de los insecticidas evaluados más el costo de aplicación de insecticidas. Para obtener los costos fijos se incluye la depreciación de equipos usados, costos de insumos usados, mano de obra, control de plagas y enfermedades etc. Al obtener el beneficio neto, se restó los costos variables menos los costos fijos de cada tratamiento respectivamente (CIMMYT, 1988).

Costos variables por parcela. Son todos aquellos costos por unidad de área relacionados con los insumos comprados, labores mecánicas, maquinaria, etc., que varían de un tratamiento a otro.

Costos totales por parcela: Es la suma de los costos fijos y los costos variables.

Rendimiento bruto: La producción de cada uno de los tratamientos por unidad área.

Rendimiento ajustado: Es el rendimiento bruto reducido en un determinado porcentaje, con el propósito de reflejar la diferencia entre el rendimiento experimental y el que podría ser obtenido por el productor con ese mismo tratamiento.

Precio del producto: es la relación de cambio por dinero, es el número de unidades monetarias que se necesitan para obtener a cambio una unidad de producto.

Beneficio bruto: El rendimiento ajustado de cada uno de los tratamientos, por el precio unitario del producto del mercado.

Beneficio neto: Es la diferencia entre el ingreso bruto total y los costos totales de producción.

Análisis de dominancia: Este análisis se efectuó ordenando los costos variables de cada tratamientos de menores a mayores, se dice que un tratamientos es dominado cuando sus 
beneficios netos son menores o iguales a los de un tratamiento que tiene costos que varían más bajos Análisis de la tasa retorno marginal (CIMMYT, 1988).

Tasa de retorno marginal (TRM). Según CIMMYT (1988) es un procedimiento que se utiliza para calcular las tasas de retorno marginal entre los tratamientos no dominados comenzando con el tratamiento de menor costo y procediendo paso a paso a los que les siguen en escala ascendente. Se calculó mediante la fórmula: $\mathrm{TRM}=$ Beneficio marginal $\div$ Costo marginal $\times 100$

Análisis estadísticos. Una vez recolectados los datos en campo se procedió a ordenarlos por variable para luego proceder a hacer un análisis de varianza ANDEVA (PROC GLM en SAS, 2003.V.9.1). Se realizó una separación de medias por Duncan $(P=0.05)$. Luego se hicieron comparaciones en los rendimientos de cada tratamiento y se determinó la rentabilidad de los tratamientos sometiendo los datos a un análisis económico de las variables agronómicas mediante un análisis de presupuesto parcial a través de la metodología del CIMMYT (1988).

\section{RESULTADOS Y DISCUSIÓN}

Fluctuación poblacional del ácaro blanco. Se comparó la fluctuación poblacional del ácaro blanco en el cultivo de la chiltoma en el Municipio de Tisma desde los siete días después del trasplante hasta los 78 ddt. El análisis de varianza realizado de la fluctuación poblacional del ácaro blanco indica que existe diferencia significativa entre los tratamientos $(P=0.017)$, donde el tratamiento Madero Negro refleja las poblaciones más bajas con 1.68 ácaros por planta, seguido de Chile+Ajo+Jabón con 1.74 y Neem con 1.86 ácaros por planta, comparados con los demás tratamientos. Según Orellana y Escobar (2005), el ataque por ácaros puede ser en etapas tempranas pero es más frecuente durante la floración y fructificación. Durante todo el periodo de crecimiento del cultivo, se presentó el ataque del ácaro blanco. Según INTA (2004), describe que las épocas calurosas y secas favorecen a la diseminación de esta especie, por ende estas precipitaciones contribuyeron a la disminución poblacional del ácaro.

Severidad del daño de ácaro blanco. Desde los siete días después del trasplante, se presentaron los porcentajes de severidad del daño de ácaro blanco en los tratamientos evaluados. El análisis de varianza realizado del Porcentaje de severidad del daño de ácaro blanco a los 78 días después del trasplante, indica que no existe diferencia significativa entre los tratamientos, aunque los tratamientos
Chile+Ajo+Jabón, Eucalipto y Madero Negro presentaron los porcentajes más bajos con $84.33,84.5$ y $84.75 \%$, respectivamente. Durante el manejo del ensayo se observó que la variedad Natalie es altamente susceptible a las toxinas transmitidas por el acaro blanco, teniendo como consecuencia que sus brotes nuevos no se desarrollen rápidamente, las toxinas se desplazan en el sistema de la planta provocando que el porcentaje de severidad del daño aumenta aun con bajas poblaciones de ácaro blanco.

Cuadro 1. Promedios de ácaros por planta y porcentaje de severidad del daño

\begin{tabular}{ccc}
\hline Tratamientos & $\begin{array}{c}\text { Ácaros/planta } \\
\text { Medias } \pm \text { ES }\end{array}$ & $\begin{array}{c}\text { \% Severidad } \\
\text { Medias } \pm \mathrm{ES}\end{array}$ \\
\hline Madero Negro & $1.68 \pm 0.05 \mathrm{a}$ & $84.33 \pm 1.61$ \\
Chile+Ajo+Jabón & $1.74 \pm 0.06 \mathrm{ab}$ & $84.5 \pm 1.30$ \\
Neem & $1.86 \pm 0.12 \mathrm{ab}$ & $84.75 \pm 1.28$ \\
Crisantemo & $1.94 \pm 0.06 \mathrm{~b}$ & $85.5 \pm 1.36$ \\
Testigo & $1.96 \pm 0.06 \mathrm{bc}$ & $85.5 \pm 1.20$ \\
Eucalipto & $1.98 \pm 0.06 \mathrm{c}$ & $85.6 \pm 1.82$ \\
N & 5996 & 360 \\
SD; C.V & $2.38 ; 128.39$ & $11.22 ; 13.20$ \\
\hline F; df; P) & $(2.76 ; 5990 ; 0.017)$ & N.S
\end{tabular}

$\mathrm{ES}=$ Error estándar, $\mathrm{CV}=$ Coeficiente de Variación, $\mathrm{SD}=$ Desviación Estándar, $\mathrm{N}=$ Número de datos utilizados en el análisis, $\mathrm{F}=$ Fisher calculado, $d f=$ Grados de libertad del error, $P=$ Probabilidad según Duncan.

Comparación del rendimiento total en ( $\left.\mathrm{kg} \mathrm{ha}^{-1}\right)$. Los rendimientos totales obtenidos muestran que el tratamiento con mayor rendimiento fue la parcela tratada con Madero Negro con $2401.30 \mathrm{~kg} / \mathrm{ha}$. Los tratamientos evaluados con Chile+Ajo+Jabón, Neem y Crisantemo obtuvieron rendimientos de 2 139.21; 2117.96 y $2096.71 \mathrm{~kg} \mathrm{ha}^{-1}$ respectivamente, en cambio los tratamientos Testigo y Eucalipto obtuvieron los rendimientos un poco más bajos con 1933.80 y $1533.57 \mathrm{~kg}$ ha $^{-1}$ respectivamente.

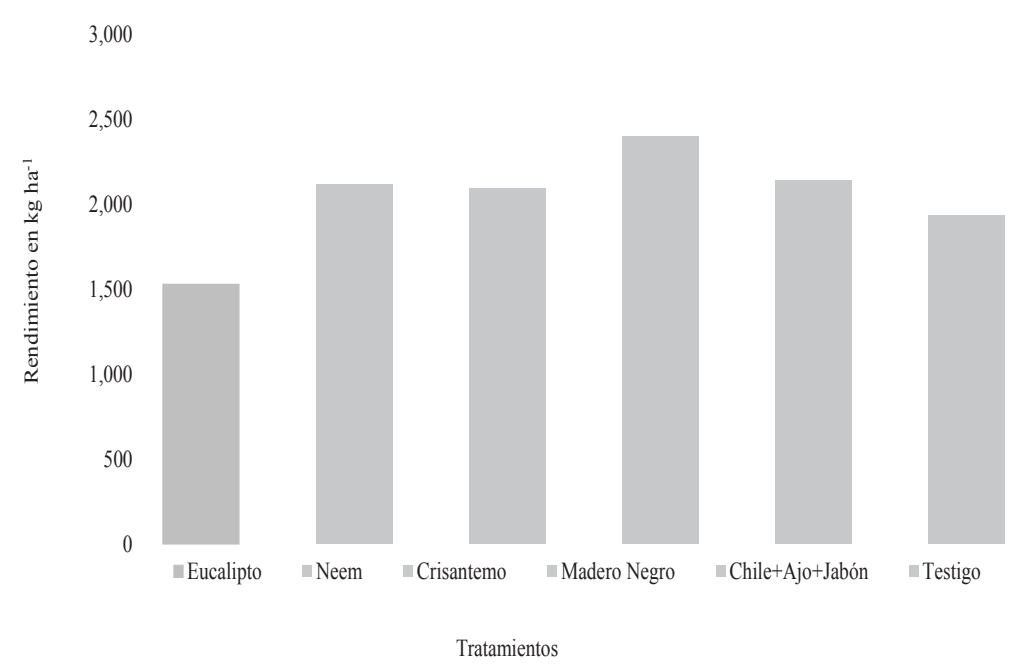

Figura 1. Comparación del rendimiento total en $\mathrm{kg} / \mathrm{ha}$ de chiltoma por tratamiento evaluado, en el periodo entre Septiembre a Noviembre 2014, Tisma, Masaya. 
Comparación económica de los tratamientos

Presupuesto parcial. EL análisis de presupuesto parcial realizado según la metodología CIMMYT (1988) determinó que los mayores costos variables los obtuvieron los tratamientos Neem y Crisantemo con 167 y 77 USD/ha, en cambio los que presentaron menores costos variables fueron Madero Negro y Eucalipto ambos con 10.8 USD/ ha seguido de testigo y Chile+Ajo+Jabón con 3.2 y $8.54 \mathrm{USD} / \mathrm{ha}$.

EL tratamiento que obtuvo el mayor beneficio neto fue el Madero Negro con 3 164.53 USD/ ha en cambio el tratamiento que presentó los menores beneficios netos fue el Eucalipto con 1680.72 USD/ha.

Cuadro 2. Presupuesto Parcial

\begin{tabular}{|c|c|c|c|c|c|c|}
\hline Concepto & Eucalipto & Neem & Crisantemo & $\begin{array}{l}\text { Madero } \\
\text { Negro }\end{array}$ & Chile+Ajo+Jabón & Testigo \\
\hline $\begin{array}{l}\text { Rendimiento } \\
\text { en } \mathrm{kg} \mathrm{ha}^{-1}\end{array}$ & 1522.20 & 2102.2 & 2081.15 & 2383.4 & 2123.34 & 1919.4 \\
\hline $\begin{array}{l}\text { Rendimiento } \\
\text { ajustado }(10 \%) \\
\quad\left(\mathrm{kg} \mathrm{ha}^{-1}\right)\end{array}$ & 1369.98 & 1892.0 & 1873.04 & 2145.1 & 1911.01 & 1727.3 \\
\hline $\begin{array}{l}\text { Precio de cam- } \\
\text { po (USD) }\end{array}$ & 1.90 & 1.90 & 1.90 & 1.90 & 1.90 & 1.90 \\
\hline $\begin{array}{l}\text { Ingreso bruto } \\
\text { (USD) }\end{array}$ & 2602.96 & 3594.8 & 3558.77 & 4075.7 & 3630.92 & 3281.8 \\
\hline $\begin{array}{c}\text { Total Costos } \\
\text { Variables USD } \\
\text { ha }^{-1}\end{array}$ & 10.71 & 106.19 & 737.48 & 10.71 & 8.46 & 3.17 \\
\hline $\begin{array}{l}\text { Total de costos } \\
\text { fijos USD ha }\end{array}$ & 1213.93 & 1213.9 & 1213.93 & 1213.9 & 1213.93 & 1213.9 \\
\hline $\begin{array}{l}\text { Costo total de } \\
\text { producción } \\
\text { USD ha-1 }\end{array}$ & 1242.42 & 1320.1 & 1951.41 & 1224.6 & 1222.39 & 1217.1 \\
\hline $\begin{array}{c}\text { Beneficio neto } \\
\text { USD ha }\end{array}$ & 1360.54 & 2274.7 & 1607.36 & 2851.1 & 2408.53 & 2064.7 \\
\hline
\end{tabular}

Precio oficial del dólar a febrero 2015: C\$26.76 fuente: BCN. Precio del producto en campo al momento de la cosecha: $1.90 \mathrm{USD} / \mathrm{kg}$

El presupuesto parcial es un método que se utiliza para organizar los datos experimentales con el fin de obtener los costos y los beneficios de los tratamientos evaluados. En el análisis se utilizan únicamente los costos que varían de un tratamiento a otro. Por lo tanto el proceso de aplicación de este enfoque debe generar una recomendación para los agricultores (CIMMYT, 1988).

Análisis de dominancia. El análisis de dominancia se utiliza para tratamientos que en términos de ganancia ofrecen la posibilidad de ser escogidos para recomendarse a los agricultores. Este análisis determina que tratamiento domina en cuanto a beneficios netos y costos variables (CIMMYT, 1988).
Cuadro 3. Análisis de dominancia

\begin{tabular}{cccc}
\hline Tratamiento & $\begin{array}{c}\text { Costo Variable } \\
\text { USD/ha }\end{array}$ & $\begin{array}{c}\text { Beneficio Neto } \\
\text { USD/ha }\end{array}$ & Resultado \\
Testigo & 3.17 & 2064.79 & ND \\
Chile+Ajo+Jabón & 8.46 & 2408.53 & ND \\
Eucalipto & 10.71 & 1360.54 & $\mathrm{D}$ \\
Madero Negro & 10.71 & 2851.12 & ND \\
Neem & 106.19 & 2274.71 & $\mathrm{D}$ \\
\hline Crisantemo & 737.48 & 1607.36 & $\mathrm{D}$ \\
\hline
\end{tabular}

El análisis de dominancia realizado a este estudio refleja que los tratamientos Eucalipto, Crisantemo y Neem resultaron ser dominados, esto se debe a que presentan menores beneficios netos y mayores costos variables que el resto de los tratamientos incluidos en este estudio, por lo tanto, estos fueron excluidos para la realización del análisis de la tasa de retorno marginal. Los tratamientos Testigo, Chile+Ajo+Jabón resultaron ser no dominados, por lo tanto son los que toman en cuenta para realizar el análisis de la tasa de retorno marginal.

Análisis de la tasa de retorno marginal (TRM). El análisis de retorno marginal indica lo que el agricultor puede ganar en promedio con su inversión cuando decide cambiar una práctica por otra más rentable, sin embargo no se puede tomar una decisión rápida con respecto a un tratamiento sin haber determinado la tasa de retorno que sería la decisión del agricultor. Según CIMMYT (1988), la tasa de retorno marginal mínima aceptable para el agricultor es de 50 y $100 \%$. El análisis de la tasa de retorno marginal refleja que para el control de ácaro blanco el mejor tratamiento es el Madero Negro ya que por cada dólar invertido el productor obtiene una tasa de retorno marginal de $19670.66 \%$, es decir que por cada dólar invertido obtiene 196.70 dólares adicionales, por otro lado si el productor decide usar Chile $+\mathrm{Ajo}+\mathrm{Ja}-$ bón para el control de ácaro blanco por cada dólar invertido obtiene una tasa de retorno marginal de $6497.92 \%$ lo cual equivale a 64.98 dólares adicionales una vez recuperado el dólar invertido. 
Cuadro 4. Análisis de la tasa de retorno marginal

\begin{tabular}{cccccc}
\hline Tratamientos & $\begin{array}{c}\text { Costo Variable } \\
\text { USD/ha }\end{array}$ & $\begin{array}{c}\text { Costo Marginal } \\
\text { USD/ha }\end{array}$ & $\begin{array}{c}\text { Beneficio Neto } \\
\text { USD/ha }\end{array}$ & $\begin{array}{c}\text { Beneficio Mar- } \\
\text { ginal USD/ha }\end{array}$ & $\begin{array}{c}\text { Tasa de retorno } \\
\text { marginal \% }\end{array}$ \\
\hline Testigo & 3.17 & & 2064.79 & & \\
Chile+Ajo+Jabón & 8.46 & 5.29 & 2408.53 & 343.74 & 6497.92 \\
Madero Negro & 10.71 & 2.25 & 2851.12 & 442.59 & 19670.66 \\
\hline
\end{tabular}

\section{CONCLUSIONES}

Los resultados obtenidos en el estudio determinan que los tratamientos Madero Negro y chile+ajo+jabón presentaron el mejor efecto de control del ácaro blanco.

Los mejores rendimientos comerciales lo obtuvieron los tratamientos madero negro y chile+ajo+jabón.

El tratamiento Madero Negro presentó los mayores ingresos brutos/ha, por otro lado los tratamientos Testigo y Eucalipto presentaron menores costos variables.

El tratamiento Madero Negro tuvo una tasa de retorno marginal de $19670.66 \%$ lo que quiere decir que por cada dó- lar invertido se obtiene una ganancia de 196.70 dólares netos adicionales y si usa Chile+Ajo+Jabón se obtiene una tasa de retorno de $6497.92 \%$ es decir una ganancia de 64.98 dólares netos adicionales.

\section{AGRADECIMIENTO}

Los autores de esta investigación agradecen a la Sra. Elizabeth González y al Sr. Anuar González, productores de Tisma, Masaya por prestarnos su finca donde se realizó la investigación, a la Universidad Nacional Agraria (UNA) por la financiación económica de este estudio.

\section{REFERENCIAS BIBLIOGRÁFICAS}

Aguirre, V; Delgado, V. 2010. Pesticidas naturales y sintéticos. Revista Ciencia. 13(1):43-53.

AMUNIC (Asociación de Municipios de Nicaragua). 2005. Municipios: Caracterización de municipios de Masaya (en línea). Managua, NI. Consultado 15 oct. 2014. Disponible en http//www.amunic.org/

Barberena, J; Lacayo, Y. 2011. Evaluación de alternativas botánicas y química para el manejo de ácaro blanco (Poliphagotarsonemus latus, Bank.) (Acarina: Tarsonemidae) e insectos plagas en el cultivo de chiltoma (Capsicum annuum L.), Tesis Ing., U.N.A. Managua. NI. 55 p.

CATIE (Centro Agronómico Tropical de Investigación y Enseñanza), 1993. Guía para el manejo integrado de plagas del cultivo del chile dulce. Centro Agronómico Tropical de Investigación y Enseñanza. Programa de mejoramiento del cultivo. MIP. Turrialba, CR.168P.

CIMMYT (Centro Internacional de Mejoramiento de Maíz y Trigo). 1988. La formulación de recomendaciones a partir de datos agronómicos: Un manual metodológico de evaluación económico. Edición completamente revisada. D.F. MX. 79 p.

González, J; Obregón, H. 2007. Evaluación de alternativas de protección física y química De semilleros de chiltoma (Capsicum annum L.) Contra el Ataque del complejo mosca blanca (Bemisia tabaci, Gennadius) - geminivirus. Tesis Ing. Sistemas de Protección Agrícola y Forestal. UNA, Managua, NI. 69 p.

INTA (Instituto Nicaragüense de Tecnología Agropecuaria). 2006. Guía tecnológica de chiltoma. Managua, NI. 44 p.

INTA (Instituto Nicaragüense de Tecnología Agropecuaria). 2004. Manejo integrado de plagas. Cultivo de la chiltoma. Managua, NI. 32 p.

Jiménez Martínez, E; Varela G. 2013. Módulo práctico: manejo integrado de plagas. UNA. Managua, NI, 61 p.

Jiménez Martínez, E; Martínez, R; Jirón, M. (2013). Plaguicidas botánicos y quimicos para el control del ácaro blanco (Polyphagotarsonemus latus Bank) (Acarina: Tarsonimidae) en chiltoma (Capsicum annuum L.), Tisma, Masaya. Revista Cientifica La Calera Vol. 13. No 20, p. 9-15

Lanuza, E; Rizo, E. 2012. Evaluación de productos botánicos y químicos sobre el complejo mosca blanca (Bemisia tabaci Gennadius)- Geminivirus en el cultivo de tomate (Solanum esculentum, Mill.), en Tisma- Masaya. TESIS Ing., UNA, Managua, NI. 59 P.

Marrón de Jones, R. 1983. Respuesta reproductiva del amplio acaro (Poliphagotarsonemus latus, Bank), a los regímenes constantes de temperatura humedad. s.n.t. p. 446-469.

Martínez, R; Jirón, M. 2011. Evaluación de productos botánicos y químicos para el manejo del ácaro blanco (Poliphagotarsonemus latus, Bank.) y otras plagas claves en el cultivo de chiltoma (Capsicum annuum L.) y su efecto en los enemigos naturales en Tisma, Masaya. Tesis Ing., U.N.A. Managua, NI. 47 p.

Ochoa, R.; Aguilar, H.; Vargas, C. 1991. Ácaros fitófagos de América Central. Guía ilustrada. CATIE, Turrialba, CR. 251 p. 
Orellana, H; Escobar, J. 2005. Guía técnica de cultivo de chile dulce. CENTA. La Libertad, SV. 51p.

Sevilla, E; Rodríguez, E. 2009. Evaluación de alternativas químicas y botánicas para el manejo del acaro blanco (Poliphagotarsonemus latus, Bank) en chiltoma (Capsicum annuum L.), Tisma, Masaya. Tesis Ing., U.N.A. Managua, NI. 40p.

Syngenta. 2015. Pimentón Híbrido Nathalie Syngenta (en linea). Consultado 20 Abr 2015. Disponible en http://www.granex.com. ve/productos/productos/mostrar/idProducto/8/Piment $\% \mathrm{C3} \% \mathrm{~B} 3 \mathrm{n} \% 20 \mathrm{H} \% \mathrm{C3} \%$ ADbrido $\% 20 \mathrm{Nathalie} \% 20$ Syngenta//idCatego$\mathrm{ria} / 3$

Vanderplank, JE. 1963. Plant diseases: epidemilogy and control. New York. Academia press. 69 p. 